\title{
Age Differences Between Children and Young Adults in the Dynamics of Dual-Task Prioritization: Body (Balance) Versus Mind (Memory)
}

\author{
Sabine Schaefer \\ Max Planck Institute for Human Development \\ Ulman Lindenberger \\ Max Planck Institute for Human Development
}

\author{
Ralf Th. Krampe \\ Katholieke Universiteit Leuven \\ Paul B. Baltes \\ Max Planck Institute for Human Development \\ and University of Virginia
}

\begin{abstract}
Task prioritization can lead to trade-off patterns in dual-task situations. The authors compared dual-task performances in 9- and 11-year-old children and young adults performing a cognitive task and a motor task concurrently. The motor task required balancing on an ankle-disc board. Two cognitive tasks measured working memory and episodic memory at difficulty levels individually adjusted during the course of extensive training. Adults showed performance decrements in both task domains under dual-task conditions. In contrast, children showed decrements only in the cognitive tasks but actually swayed less under dual-task than under single-task conditions and continued to reduce their body sway even when instructed to focus on the cognitive task. The authors argue that children perform closer to their stability boundaries in the balance task and therefore prioritize protection of their balance under dual-task conditions.
\end{abstract}

Keywords: dual task, children, young adults, postural stability, task prioritization

Everyday life involves numerous situations in which a motor task and a cognitive task are performed simultaneously, for example when riding a bike while being engaged in a conversation. At first sight, such dual-task situations seem easy to manage. However, when approaching a busy street intersection, one might interrupt the conversation to pay attention exclusively to traffic, temporarily prioritizing one task at the expense of the other. The need to prioritize might arise from difficulties in task coordination or from insufficient resources to attend to both tasks at the same time, both capacities being subject to developmental change. This study investigated differences between children and young adults in their ability to coordinate concurrent balance and memory performances.

Sabine Schaefer and Ulman Lindenberger, Department of Lifespan Psychology, Max Planck Institute for Human Development, Berlin, Germany; Ralf Th. Krampe, Department of Psychology, Katholieke Universiteit Leuven, Leuven, Belgium; Paul B. Baltes, Department of Lifespan Psychology, Max Planck Institute for Human Development, Berlin, Germany, and Department of Psychology, University of Virginia, Charlottesville, VA.

Ralf Th. Krampe is now at the Department of Psychology, Centrum voor Ontwikkelingspsychologie, Leuven, Belgium. Paul B. Baltes died in November 2006.

We thank Nina Blaskewitz, Stefanie Dabrowski, Gabi Faust, Anna Gronostaj, Simone Ränker, Sarah Risse, Gabi Schmid, Nina Smolarz, and Michael Zeschky for their help with data collection and Julia Delius for her helpful comments.

Correspondence concerning this article should be addressed to Sabine Schaefer, Department of Lifespan Psychology, Max Planck Institute for Human Development, Lentzeallee 94, Berlin D-14195, Germany. E-mail: schaefer@mpib-berlin.mpg.de
When a motor task and a cognitive task have to be performed concurrently, not paying sufficient attention to the motor domain may lead to falls that can have severe consequences. According to the Safe Kids U.S.A. Campaign (2007), falls are the leading cause of unintentional injury for children, and there seem to be marked developmental changes in children's abilities to recognize potentially dangerous situations and to deal with them. At the same time, risk taking in the motor domain must be considered a prerequisite for mastering motor skills, since it is impossible to learn to walk or ride a bike without falling.

The conceptual foundation for investigating dual- and multi-task performance rests on the assumption of limited resources (Wickens, 1991). Navon (1984) defined resources as any internal input essential for processing that is available in limited quantities at any point in time. Performance is expected to deteriorate in a dual-task situation if there are fewer resources available for performance of the two tasks than are required. Theories differ in whether the resources are assumed to be a single, general-purpose unit or structure (Kahneman, 1973) or whether multiple resources are assumed, such as modalities of stimulus input or response modes (Navon \& Gopher, 1979; Wickens, 1980).

Dual-task studies have often asked participants to keep up their performance in a primary task of interest while concurrently performing a secondary task. Guttentag (1989) reported that the amount of secondary-task interference declines with age during childhood, reflecting age differences in the resource demands of the primary task. In addition, children's ability to flexibly allocate resources according to instruction increases with advancing age (Birch, 1978; Irwin-Chase \& Burns, 2000).

Over the last decade, there has been an increasing awareness among life span developmentalists about the relevance of cogni- 
tive resources and intelligence for all kinds of behaviors, including sensorimotor and motor functioning. It is assumed that there are age differences with respect to the amount of cognitive resources that have to be invested into sensorimotor tasks. Naturally, the investigation of this interplay requires an alternative approach, in which dual-task costs in both domains of functioning are investigated (Li, Krampe, \& Bondar, 2005; Lindenberger, Marsiske, \& Baltes, 2000). In line with the assumption of increased cognitiveresource demands for sensorimotor functions, researchers have observed pronounced performance decrements in children (see Huang \& Mercer, 2001, for a review) and older adults (Brown, Sleik, Polych, \& Gage, 2002; Lindenberger et al., 2000; Rapp, Krampe, \& Baltes, 2006; Shumway-Cook, Woollacott, Kerns, \& Baldwin, 1997) compared with young adults when a cognitive task and a motor task had to be performed concurrently.

\section{Adaptive Resource Allocation}

More recently, several studies have focused on the adaptiveness of resource allocation to competing tasks and related differences between age groups. These studies were informed by the model of selection, optimization, and compensation (SOC), originally proposed by Paul B. Baltes and Margret M. Baltes (1990; Baltes, 1997). The SOC model features three fundamental processes of mastery through task- and context-sensitive resource allocation (Baltes, 1987; Baltes, Lindenberger, \& Staudinger, 2006; Krampe $\&$ Baltes, 2003): Selection refers to the commitment to a subset of goals or functional domains, instead of distributing one's energy over many diverse areas. Optimization refers to situations in which means are applied to achieve optimal functioning or desired outcomes, and resources are allocated and refined in order to reach a certain outcome. Compensation is relevant in the management of resource loss and describes the substitution of means or the use of alternative means to maintain a given level of functioning (e.g., the use of a walking stick after an accident).

A specific form of selection in the SOC model, loss-based selection, comes into play if the maintenance of a given level of functioning is threatened. Recent studies indeed suggest that older adults are more likely to prioritize their motor performance than young adults, presumably to protect themselves from falls and resulting physical harm (Li, Lindenberger, Freund, \& Baltes, 2001; Rapp et al., 2006; Schaefer, Huxhold, \& Lindenberger, 2006). Li et al. (2001) asked young and older adults to memorize word lists while walking on a narrow track. In older adults, memory performance was more strongly reduced than in young adults, whereas performance reduction in the walking task was comparably high for both age groups. Rapp et al. (2006) found pronounced dualtask costs in postural stability and memory when older adults performed a working memory task while standing on a stable platform. In contrast, costs in balance in older adults and even patients with Alzheimer's disease were reduced to the level of young adults when the posture task was made more salient by platform movements. Lövdén, Schellenbach, Grossmann-Hutter, Krüger, and Lindenberger (2005) asked young and older men to walk on a treadmill while concurrently performing a route-learning task in a virtual maze. Supporting balance with use of a handrail improved older men's way-finding performance but not younger men's, presumably because older adults were able to invest some of the cognitive resources that they had previously invested into the treadmill-walking task into the way-finding task.

Although resource trade-offs between different functional domains play an important role in early ontogeny, related processes have hardly been studied in children. This study investigates whether, similar to older adults, children protect their postural stability in dual-task situations. To this end, we had children and young adults balance on an ankle-disc board while they concurrently performed either an episodic-memory or a working-memory task.

\section{Outline of the Study and Hypotheses}

The primary goal of the present study was to investigate agerelated differences in how children and young adults allocate resources when performing a cognitive task and a motor task concurrently. Following the ecological approach to dual-task research (Li et al., 2005), we used combinations of tasks that mimic everyday processing demands, we considered dual-task related performance decrements for all component tasks, we manipulated task difficulties at the individual level, and we implemented a differential-emphasis condition (see also Lindenberger et al., 2000). Balancing on the ankle-disc board was the sensorimotor task in the present study, since postural control is particularly sensitive to shifts in resource allocation between two concurrent tasks (e.g., Rapp et al., 2006). We used two different cognitive tasks - the method of loci (MOL) task to assess episodic memory and the N-back task as a measure of working memory-and predicted that patterns of dual-task performance would generalize across these two cognitive tasks.

Differences between children and young adults in the three component skills of postural control (DeOreo \& Wade, 1971; Sheldon, 1963; Woollacott \& Shumway-Cook, 1990), episodic memory (Brehmer, Li, Müller, von Oertzen, \& Lindenberger, 2007; Harnishfeger \& Bjorklund, 1990), and working memory (Craik \& Bialystok, 2006; Hitch, Towse, \& Hutton, 2001) are well documented in the literature. To ensure that participants could make maximal use of their potential, we trained them extensively in each component task prior to the dual-task assessments. Task difficulties of the cognitive tasks were adjusted individually to equalize the amount of cognitive resources needed under singletask conditions. Our motor task-balancing on the ankle-disc board on a stable or moving platform-provided a considerable challenge in the first place, and difficulty levels were not individually adjusted but fixed for all participants.

One basic assumption of the present study was that childrenlike older adults- have fewer resources available. In this context, we defined resources as any person-specific skill or ability that enabled the individual to perform well on the tasks of interest Thus, we expected lower levels of performance in children compared with such levels in young adults under single-task conditions at identical levels of difficulty. Concerning the dual-task situation, we expected performance decrements (dual-task costs) for all three age groups. According to the SOC model (Baltes \& Baltes, 1990), selection processes can influence the trade-off pattern between two task domains, with higher dual-task costs in one task as opposed to the other. We hypothesized that children would show such a trade-off pattern in favor of the balance task. Unpublished previous findings from our laboratory showed that children sway more than 
adults when balancing on the ankle-disc board (see also Lippens, 2005) and therefore already operate closer to their stability boundaries under single-task conditions. In a dual-task situation, they were expected to preserve a sufficiently large safety region for maintaining their postural stability by focusing their attention on the motor domain. Young adults, on the other hand, were expected to show a more even distribution of dual-task costs across the two domains, without significant differences between cognition and balance.

In the dual task of the final session, participants were instructed to focus more strongly on one task than on the other, either by further reducing their body sway or by achieving consistently high scores in the cognitive task. We predicted higher flexibility in young adults, given their advantage in overall resources and their larger safety regions in the balance task. Due to the importance to children of maintaining their balance when resources are taxed, we expected them to preserve their described prioritization pattern of loss-based selection.

\section{Method}

\section{Participants}

Three age groups were tested in this study: 9-year-old children $(M=9.34, S D=0.27), 11$-year-old children $(M=11.49, S D=$ $0.18)$, and young adults between 20 and 25 years of age $(M=$ $22.91, S D=1.34)$. In each age group, 4 males and 5 females were tested. Participants were drawn from the subject pool of the Max Planck Institute for Human Development in Berlin. Young adults were college or university students, and children attended local elementary schools. The groups of subjects were comparable in social class. All participants were German citizens. Some of the children had one parent who had immigrated to Germany, but German was the first language spoken at home in all families. Participants were tested individually and received 10 euros per session for their participation. The institute's ethics committee approved the study.

People with illnesses that could affect their motor performance (neurological disorders such as cerebral palsy or brain tumors, acute or chronic ear infections, diabetes, vestibular disorders, injuries of the legs or ankle joints, and attention-deficit/ hyperactivity syndrome) were excluded from participation. Participants' performance on the digit symbol substitution test measuring cognitive speed, the digit span forward and backward test measuring memory span, and the word meanings test measuring verbal knowledge from the children's or adults version of the Wechsler Intelligence Scale were between the 50th and the 100th percentile in comparison with published age norms (Tewes, 1991; Tewes, Rossmann, \& Schallberger, 1999). The overall picture on these measures was consistent with the developmental literature showing that cognitive speed and working memory improve during childhood and do not reach mature levels of performance before late adolescence or young adulthood (e.g., Case, Kurland, \& Goldberg, 1982; Fry \& Hale, 1996; Kail, 1991).

\section{Apparatus}

A Power Macintosh 7100/ 80 computer (Apple, Inc., Cupertino, CA) was used to present the stimuli of the two cognitive tasks under single-task conditions and the baseline condition for the balance task. In the dual-task condition, stimuli were presented on a Pentium III personal computer (Intel, Santa Clara, CA) on a 22-inch (56-cm) Sony monitor (Sony Corp., Tokyo, Japan).

The balance task consisted of balancing on a so-called ankledisc board or wobble board. On the board, participants stood on a circle (diameter $=39 \mathrm{~cm}$ ) with a special corrugated surface to prevent their feet from sliding off. The lower part of the board was convex and $8 \mathrm{~cm}$ high. The board was placed on a $40 \mathrm{~cm} \times 60 \mathrm{~cm}$ dynamic force platform (Kistler force platform 9286AA, Kistler Instrumenten, Winterthur, Switzerland), surrounded by a second $120 \mathrm{~cm} \times 140 \mathrm{~cm}$ platform with safety handrails. People were additionally secured by safety belts used in mountain climbing (Liberty Adjust Tape D, Mammut, Cologne, Germany). While balancing, participants faced a computer monitor on the left-hand side of the platform. The height of the monitor could be adjusted individually to eye level.

The measurement device for balance consisted of the balance platform and two computers. The measurement computer ( $\mu-\mathrm{M}-\mathrm{S}$ Eth-RJ45, mcm Prüfsysteme, Berlin, Germany) controlled the speed and angle of platform movement and collected data from 12 sensors. The other computer (NEXOS Pentium III, $5000 \mathrm{MHz}$, $\mathrm{PC} / \mathrm{NT}$ ) provided feedback on balance performance and presented the cognitive stimuli during dual-task trials. Auditory stimuli were presented over headphones.

The dynamic force platform had 12 piezoelectric sensors, 3 in each corner. It measured postural sway by recording the components of the ground reaction force on the lateral, vertical, and anterior-posterior horizontal axes, along with the three respective moment components. Signals were collected at $1000 \mathrm{~Hz}$ and were then software-filtered with a Butterworth third-order low-pass filter $(20 \mathrm{~Hz})$ to remove high-frequency artifacts. The variable of interest in the current study was the area within which the body's center of pressure (COP) moved in a given time, representing the portion of the base of support used while participants were balancing on the board. Good performance, which is achieved through small and well-coordinated body movements, results in small COP areas (Manckoundia, Pfitzenmeyer, d'Athis, Dubost, \& Mourey, 2006; Marchese, Bove, \& Abbruzzese, 2003). ${ }^{1}$

Balance performance was assessed on the stable and moving platforms. For trials on the moving platform, a central robotic axis (Power Cube Rotari PR 110, mcm Prüfsysteme, Berlin, Germany) produced a triangular wave of continuous movement along the lateral plane at a frequency of $0.5 \mathrm{~Hz}$ and with an angle of $3^{\circ}$. Participants had to counteract the movement of the platform by shifting their weight in the opposite direction. Platform movements led to larger COP areas than balancing on the stable platform.

\footnotetext{
${ }^{1}$ We decided to use the center of pressure (COP) area instead of alternative measures like mean velocity of the COP or root-mean-square distance. We considered the COP area measure to be most comparable across the two different platform movement conditions that have been used, with the stable platform condition resulting in a rather round COP area and the moving platform condition resulting in an elliptically shaped area. In addition, we assumed that the COP area most accurately reflects whether people are approaching the limits of their stability.
} 


\section{Experimental Tasks and Stimuli}

Method of loci (MOL) memory task. The MOL task required participants to memorize a list of words presented auditorily. Participants were instructed in the MOL strategy, which involves the use of a highly familiar ordered sequence of mental-map locations as a structure for encoding and retrieving new information (Bower, 1970; Lindenberger, Kliegl, \& Baltes, 1992). Participants formed interactive images connecting to-be-remembered items to the locations of the mental map. Instruction encouraged the creation of images that were interactive and dynamic and that included different sensory dimensions (e.g., smells, sounds, and movement). For example, if the location cue was "bed" and the to-be-remembered word was "frog," one could imagine a frog sitting on one's bed, or jumping up and down (movement) and croaking (sound). During recall, participants revisited the locations of their mental map and tried to retrieve the items imagined. All participants worked on a 20-item list of locations of objects that can usually be found in most households (e.g., bed, desk, chair, television, refrigerator, and so on). Location cues always appeared in exactly the same order and were presented for encoding and retrieval. Since list length varied within and between participants (discussed in the Procedure section), each new list started at the next location of the location list.

We used 40 word lists per participant. All the words used in the study were highly imaginable and highly concrete nouns stemming from the word pool used by Singer, Lindenberger, and Baltes (2003). For the first three sessions of the study, separate lists with nonrandom pairings of locations and words were constructed. For the following sessions, 972 words from the word pool used by Singer et al. (2003) were used for the young adults, and a subset of 429 of these words that had been shown to be familiar to children were used for that age group. Words were drawn randomly from the word pool without replacement. Note that although the location cues were repeated over the course of the study, participants had to construct new mental images each time in order to remember the words.

Time frames for the auditory presentation of the locations were fixed to 1,200 ms. During encoding, participants either sat in front of the computer (single-task condition) or stood on the ankle-disc board (dual-task condition). At recall, participants tried to retrieve the to-be-learned items from memory, starting with the first location cue of the list and subsequently working on the remaining locations of the list one after the other. The upper time limit for each answer was $90 \mathrm{~s}$. Participants received feedback about their performance at the end of each list.

$\mathrm{N}$-back working memory task. The N-back task requires monitoring, short-term storage, and scheduled retrieval of digits and is assumed to rely heavily on working memory functioning and executive control processes (Dobbs \& Rule, 1989; Smith \& Jonides, 1999). In the current study, N-back stimuli were digits from 1 to 9 presented in succession in a white square $(8 \mathrm{~cm} \times 8$ $\mathrm{cm}$ ) on a black background on the computer screen. Digits were about $6 \mathrm{~cm}$ high and $4 \mathrm{~cm}$ wide and were drawn randomly for presentation, with the constraint that at least 2 different digits had to be presented before a certain digit could be repeated. Furthermore, numerically adjacent digits never appeared in succession.

Depending on the condition, participants had to read each digit aloud as soon as it appeared (N-back 0), or they had to postpone the verbalization of the digit until the second-next digit was presented (N-back 2 for children) or until the fourth digit in the series was shown (N-back 4 for young adults). Items were scored as correct if the correct digit was named in the correct time window. Errors were registered (a) if a digit was named that was not correct in that serial position, (b) if no digit was named at all, or (c) if a digit was named earlier than it should have been named. Correct digits were only scored to the second error within a trial. Inter-stimulus intervals (ISIs) and number of successively presented digits varied according to the phase of the study.

Balance task. Participants were instructed to "sway as little as possible" on the ankle-disc board, with their arms loosely at their sides. Feedback about the COP movement was given after each trial.

Two different single-task (or baseline) conditions were used for the balance task. To control for the influence of load-independent factors like verbalization and auditory stimulus perception, we asked the participants to perform the balance task while concurrently listening to a stream of color words and animal voices as a single-task (baseline) condition for balancing while performing the MOL task. Participants were asked to detect three different animal voices (a dog barking, a cow mooing, or a goat bleating) in a stream of color words. Trials for balance in combination with the MOL task or the animal-voice task always lasted $60 \mathrm{~s}$. The single-task (baseline) condition for balancing while performing the $\mathrm{N}$-back task required participants to simply read digits presented on the computer screen aloud during the balance trial (N-back 0). Trials for balance in combination with the N-back task always lasted for $35 \mathrm{~s}$. The number of stimuli presented in the animalvoice or the N-back task and the ISIs between successive stimuli corresponded to the task parameters that a participant encountered in the cognitive task (MOL/N-back) during that study phase.

\section{Procedure}

The study consisted of nine sessions each lasting for $1 \mathrm{hr}$. Participants were tested individually, with two experimenters present at all times. Table 1 presents an overview on the study design. In the first three sessions, participants were instructed and trained in the three component tasks under single-task conditions. In the adaptive phase, consisting of Sessions 4 and 5, we manipulated task difficulty of the cognitive tasks by varying list length and ISI concurrently. High performance levels in previous trials led to more items and shorter ISIs in the following trial; with this manipulation, we sought to adjust each individual's performance to the level of about $80 \%$ correct. For the MOL task, list length could vary between 6 items (ISI $=8,500 \mathrm{~ms}$ ) and 20 items (ISI $=$ $1,600 \mathrm{~ms}$ ). N-back trials could vary between 13 items (ISI $=2,500$ $\mathrm{ms}$ ) and 32 items (ISI $=1,000 \mathrm{~ms}$ ). This procedure guaranteed that overall trial length remained the same for each task over the course of the study. Furthermore, during the adaptive phase, participants were trained in balancing on the board while performing the cognitively undemanding baseline task (reading out numbers or listening to animal voices). The assessment of dual-task performance took place in Sessions 6-8, in which N-back performance or MOL performance was measured while participants were concurrently balancing on the ankle-disc board. Participants were instructed to perform both tasks concurrently "as well as possible." Different difficulty levels of the balance task (stable or moving platform) were tested in one session each, and the order of these 
Table 1

Overview of the Study Design

\begin{tabular}{|c|c|c|c|}
\hline \multirow[b]{2}{*}{ Study session } & \multicolumn{3}{|r|}{ Task } \\
\hline & Method of loci & N-back & Balance \\
\hline 1 & Pretest & Training & Stable platform \\
\hline 2 & Instruction & Training & Stable platform \\
\hline 3 & Training & Training & Stable and moving platforms \\
\hline 4 and 5 & Adaptive training $^{\mathrm{a}}$ & Adaptive training $^{\mathrm{a}}$ & Stable and moving platforms with secondary tasks \\
\hline $6-8$ & Single- and dual-ta & assessment with di & fferent difficulty levels of the balance task \\
\hline 9 & Single- and dual-ta & assessment with di & fferential-emphasis instruction \\
\hline
\end{tabular}

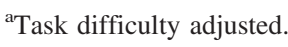

sessions was counterbalanced across participants. ${ }^{2}$ At the beginning and end of each dual-task session, single-task performance for each component task was reassessed in order to establish a reliable single-task baseline. In the last session, dual-task performance was measured under differential-emphasis instructions. Participants were balancing on the stable platform while concurrently performing the N-back task. There were three different instruction conditions: (a) "Focus more on the N-back task," (b) "Focus more on the balance task," or (c) "Focus on both tasks equally," the order of which was counterbalanced across participants. Participants were reinforced for successfully reducing their dual-task costs in the domain of emphasis by a system of credit points that could be traded in for candy.

\section{Data Processing and Statistical Analyses}

To compare performance changes from single- to dual-task conditions across different domains (cognition and balance) and cognitive tasks (MOL and N-back), we calculated proportional dual-task costs (DTCs; Somberg \& Salthouse, 1982). Proportional DTCs express in percentages the performance reductions of each individual's single-task performance. Positive values indicate that performance deteriorated from single- to dual-task, while negative values represent performance improvements.

The resulting DTCs were then analyzed with a mixed-design analysis of variance (ANOVA) with task modality (2; cognition vs. balance), cognitive task (2; N-back vs. MOL), and balance difficulty (2; stable vs. moving platform) as within-subjects factors, and age group (2; children vs. adults and 9-year-olds vs. 11-yearolds) as the between-subjects factor. This analysis was followed up by performance of $t$ tests.

\section{Results}

\section{Effects of Instruction and Adaptive Training and Stability of Single-Task Performance}

MOL task. In the first session, young adults showed higher memory performance than children, whereas differences between 9and 11-year-olds did not reach significance. Furthermore, performance improved in all age groups following instruction, and this improvement did not interact with the age contrasts. The adaptive procedure successfully equalized participants on task performance when performance was expressed as the percentage correct.
In the course of the dual-task phase, single-task MOL performance did not show systematic changes over several sessions. However, there was a significant performance reduction from the single-task trial assessed at the beginning of the session to the trial assessed at the end of the session, with performance being better in the first trial, probably caused by MOL-specific processes of proactive interference (cf. Kliegl \& Lindenberger, 1993). Furthermore, the age groups did not differ in their average percentage of correct performances. Reliability coefficients of the MOL singletask trials were highly significant within and across age groups throughout the dual-task phase (.96-.98).

$N$-back. Due to children and young adults working on different versions of the N-back task (N-back 2 vs. N-back 4), absolute values for N-back performance could not be compared. N-back performance expressed in percentage correct did not differ between children and young adults and between 9- and 11-year- olds at the end of the adaptive phase, and it remained stable over the course of the dual-task assessment. Reliability coefficients for $\mathrm{N}$-back single-task performance were highly significant within and between age groups, ranging from .82 to .98 .

Balance performance. In order to reduce the influence of outliers, we square-root transformed COP areas before averaging the trials of the same condition. For the third session, in which balance performance was assessed without any additional cognitive task and in which trials always lasted for $30 \mathrm{~s}$, COP areas were larger in children than in adults, $F(1,24)=20.46$, mean square error $(M S E)=390331.58, p<.01, \eta^{2}=.460$, while differences between 9 - and 11-year-olds failed to reach significance by a slight margin, $F(1,24)=3.99, M S E=390331.58, p=.057, \eta^{2}=.142$. Furthermore, COP areas were larger on the moving than on the stable platform, $F(1,24)=202.47, M S E=269696.37, p<.01$, $\eta^{2}=.894$, and this effect interacted significantly with the age contrast comparing children with young adults, $F(1,24)=17.69$, $M S E=269696.37, p<.01, \eta^{2}=.424$, while the interaction with the age contrast comparing 9- with 11-year-olds did not reach significance, $F(1,24)=1.54, M S E=269696.37, p=.226, \eta^{2}=$ .060 (cf. Fig. 1).

\footnotetext{
${ }^{2}$ An additional difficulty level in the balance task that differed depending on age group was assessed in the study. These data are not presented here.
} 


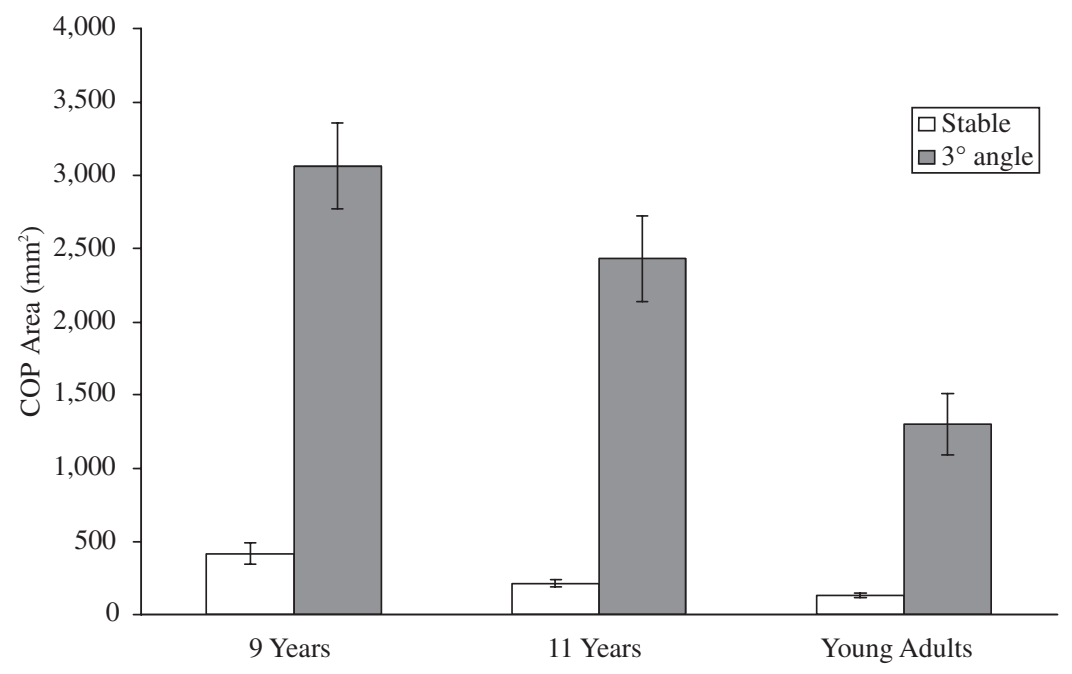

Figure 1. Children swayed more than adults, and sway increased on the moving platform $\left(3^{\circ}\right.$ angle). Error bars represent standard errors of the mean. $\mathrm{COP}=$ center of pressure.

In consecutive sessions, COP areas were larger for the trials with the secondary task of listening to animal voices as compared with reading out numbers, probably due to differences in trial duration (60 s vs. 35 s). No systematic changes in single-task balance performance over the course of the dual-task phase could be detected. Reliability coefficients for the different balance conditions within and across age groups ranged between .12 and .93 , with a median of .86 , and with 14 of the total of 16 values being highly significant. ${ }^{3}$

\section{Dual-Task Performance}

Raw data for the three tasks (MOL, N-back, and balance) are presented in Schaefer (2005). The present report focuses on the analyses of proportional DTCs. Table 2 presents the DTCs for each task domain under investigation in Sessions 6-8. A mixed-design ANOVA was conducted with task modality ( 2 ; cognition vs. balance), cognitive task (2; N-back vs. MOL), and balance difficulty ( 2 ; stable vs. moving) as within-subjects factors, and age group (2; children vs. adults and 9-year-olds vs. 11-year-olds) as the between-subjects factor. The analysis of the between-subjects effect of age group revealed no significant differences between children and young adults ( $p=$ .133 ) or between 9 - and 11-year-olds, $(p=.715)$, indicating that overall DTCs (averaged over task modality, cognitive task, and balance difficulty) did not differ between the age groups.

For the within-subjects effect of task modality, a significant main effect was detected, $F(1,24)=16.38, M S E=685.46, p<$ $.001, \eta^{2}=.406$, and there was no significant interaction of the modality effect and the age contrast comparing 9- with 11-yearolds, $F(1,24)=0.01, M S E=685.42, p=.917, \eta^{2}=.000$, whereas there was a marginally significant interaction of the modality effect and the age contrast comparing children with young adults, $F(1,24)=4.27, M S E=685.42, p=.050, \eta^{2}=$ .151. This indicates that the DTCs of the cognitive and balance domain differ in children and supports the assumption that children prioritize balance performance over cognitive performance when their balance is challenged.
The within-subjects main effect of cognitive task was not significant, $F(1,24)=0.15, M S E=439.99, p=.703, \eta^{2}=.006$, indicating that DTCs for the N-back task and DTCs for the MOL task (averaged over task modality and balance difficulty) did not differ. However, there was a significant interaction of that effect with the age contrast comparing 9- with 11-year-olds, $F(1,24)=8.11, M S E=439.99, p<$ $.05, \eta^{2}=.253$ (due to 9-year-olds showing higher DTCs for MOL than for N-back, and 11-year-olds showing the opposite pattern), and no interaction with the contrast comparing children with young adults, $F(1,24)=0.11, M S E=439.99, p=.744, \eta^{2}=.005$.

Furthermore, the within-subjects effect of balance difficulty was not significant, $F(1,24)=.05, M S E=578.94, p=.818, \eta^{2}=.002$, and it did not interact with any of the age contrasts (9-year-olds vs. 11 -year-olds, $p=.110$, and children vs. adults, $p=.516$ ). Contrary to our expectations, DTCs did not vary systematically as a function of balance-task difficulty. Specifically, we found evidence in the expected direction for the N-back task but not for the MOL task. ${ }^{4}$ These

\footnotetext{
${ }^{3}$ Reliability coefficients were calculated for the trials of each balance condition (stable platform with numbers, moving platform with numbers, stable platform with animal voices, moving platform with animal voices) separately, for each age group separately, and for all three age groups analyzed together. The following two values did not reach significance: stable platform with animal voices for the 9-year-olds $(r=.12)$ and moving platform with animal voices for the 11-year-olds $(r=.59)$.

${ }^{4}$ None of the possible two-way interactions between within-subjects factors reached significance (Task Modality $\times$ Cognitive Task: $p=.574$ Task Modality $\times$ Balance Difficulty: $p=.226$, Balance Difficulty $\times$ Cognitive Task: $p=.351$ ), and neither did the respective age contrasts of each two-way interaction. However, the three-way interaction of task modality, cognitive task, and balance difficulty was significant, $F(1,24)=$ 18.39, MSE $=98.50, p<.001, \eta^{2}=.434$, but this effect did not interact with the age contrasts (9-year-olds vs. 11-year-olds, $p=.389$, and children vs. adults, $p=.351$ ). This was caused by the influence of balance difficulty on cognitive DTCs in the N-back task but not in the MOL task.
} 
Table 2

Dual-Task Costs for the Different Tasks and Balance-Difficulty Conditions

\begin{tabular}{|c|c|c|c|c|}
\hline Task & Balance condition & 9 -year-olds & 11-year-olds & $\begin{array}{l}\text { Young } \\
\text { adults }\end{array}$ \\
\hline \multicolumn{5}{|c|}{ Method of loci } \\
\hline & \multicolumn{4}{|l|}{ Stable platform } \\
\hline & $M$ & 11.87 & 12.42 & 11.59 \\
\hline & $S D$ & 13.25 & 9.82 & 7.92 \\
\hline & \multicolumn{4}{|l|}{ Moving platform } \\
\hline & $M$ & 12.96 & 1.71 & 9.38 \\
\hline & $S D$ & 15.67 & 17.12 & 14.13 \\
\hline \multicolumn{5}{|l|}{$\mathrm{N}-$ back } \\
\hline & \multicolumn{4}{|l|}{ Stable platform } \\
\hline & $M$ & -7.42 & 9.52 & 2.01 \\
\hline & $S D$ & 6.05 & 24.07 & 25.80 \\
\hline & \multicolumn{4}{|l|}{ Moving platform } \\
\hline & $M$ & 15.43 & 17.75 & 7.38 \\
\hline & $S D$ & 25.91 & 12.53 & 26.29 \\
\hline \multicolumn{5}{|c|}{$\begin{array}{l}\text { Balance }+ \text { method } \\
\text { of loci }\end{array}$} \\
\hline & \multicolumn{4}{|l|}{ Stable platform } \\
\hline & $M$ & -10.44 & -12.74 & 4.13 \\
\hline & $S D$ & 27.67 & 22.95 & 31.05 \\
\hline & \multicolumn{4}{|l|}{ Moving platform } \\
\hline & $M$ & 0.90 & -19.20 & 2.12 \\
\hline & $S D$ & 31.81 & 19.62 & 19.35 \\
\hline \multicolumn{5}{|l|}{$\begin{array}{r}\text { Balance }+ \\
\mathrm{N}-\text { back }\end{array}$} \\
\hline & \multicolumn{4}{|l|}{ Stable platform } \\
\hline & $M$ & -17.39 & 0.76 & 9.20 \\
\hline & $S D$ & 16.92 & 29.31 & 27.69 \\
\hline & \multicolumn{4}{|l|}{ Moving platform } \\
\hline & $M$ & -16.89 & -7.76 & -1.13 \\
\hline & $S D$ & 20.98 & 17.62 & 13.65 \\
\hline
\end{tabular}

Note. The dual-task cost (DTC) metric expresses performance changes from single- to dual-task conditions as the percentage of each individual's single-task performance. Positive values for DTCs indicate that performance deteriorated from single-to dual-task conditions, while negative values indicate that performance improved under dual-task conditions. The table presents data from Sessions 6-8.

task-specific effects did not affect the overall pattern of results described in the following section.

To follow up the ANOVA results, we conducted paired-sample $t$ tests comparing cognitive DTCs with balance DTCs. The tests revealed significant differences in the two children's groups, $t(17)=4.31, p<.001$, but not in the young adults, $t(8)=0.784$, $p=.456$. Children showed a performance trade-off between cognition and balance in favor of the balance task, whereas young adults did not.

Figure 2 presents the cognitive and balance DTCs of the current study, demonstrating that children actually showed superior balance performance (i.e., less body sway) while performing a rather challenging cognitive task.

\section{Dual-Task Performance in the Differential-Emphasis Phase of the Study}

In the last session, N-back was used as the cognitive task, and participants were balancing on the stable platform. Figure 3 shows the performance pattern for the two tasks for the single-task and the two different emphasis conditions.
A mixed-design ANOVA on the N-back scores was conducted, with single- versus dual-task as within-subjects factor (3; singletask, dual-task "focus on N-back," dual-task "focus on balance"), and age group (2) as the between-subjects factor. A contrast comparing each of the dual-task conditions to the single-task condition did not reach significance, and there were no interactions with the age contrasts. In addition, children differed significantly from the young adults in their N-back performance, $F(1,24)=$ 7.56, $M S E=392.60, p<.05, \eta^{2}=.240$, while 9-year-olds did not differ from 11-year-olds, $F(1,24)=0.54, M S E=392.60, p=$ $.471, \eta^{2}=.022$. N-back performance generally did not change from single-to dual-task, independently of the differentialemphasis instruction.

Another mixed-design ANOVA was conducted for the balance task, with single- versus dual-task as within-subjects factor (3) and age group (2) as between-subjects factor. Please recall that the single-task condition for balance refers to balancing while reading out numbers. COP areas obtained after the instruction to focus on $\mathrm{N}$-back did not differ significantly from single-task balancing, $F(1$, 24) $=0.99, M S E=1787.04, p=.330, \eta^{2}=.040$, and there was no interaction with the age contrasts. However, participants improved their balance significantly under the instruction to focus on the balance task, $F(1,24)=32.35, M S E=1510.88, p<.01, \eta^{2}=$ .574 , and this effect interacted with the age contrast comparing children with adults, $F(1,24)=9.68, M S E=1510.88, p<.01$, $\eta^{2}=.287$, but not with the age contrast comparing 9- with 11 -year-olds, $F(1,24)=3.57, M S E=1510.88, p=.071, \eta^{2}=$ .129. Performance improvements were more pronounced in the children than in the young adults. Overall, adults showed smaller COP areas than children, $F(1,24)=15.89, M S E=15907.64, p<$ $.01, \eta^{2}=.398$, and 11-year-olds showed smaller COP areas than 9-year-olds, $F(1,24)=7.78, M S E=15907.64, p<.05, \eta^{2}=$ .245. In sum, children continued to sway less in the dual-task than in the single-task situation, and they were able to further reduce their body sway when instructed to focus on the balance task.

\section{Discussion}

The main finding in the present study is that children show a marked trade-off in their dual-task costs, with higher costs in the cognitive than in the motor domain (Fig. 2). In fact, children reliably improved their postural stability under dual-task conditions, while significantly reducing their performance in the memory tasks at the same time. In contrast, young adults showed comparably high performance decrements in both task domains when performing two tasks concurrently. We replicated and extended our findings under differential-emphasis conditions in that children continued to show performance improvements in the balance task even when they were instructed to focus more strongly on the cognitive domain.

We argue that children's prioritization of the balance task is an example of loss-based selection according to the SOC model (Baltes \& Baltes, 1990). To avoid putting their balance at risk when overall attentional demands increase (i.e., in the dual-task situation), children invest more resources into the balance task to preserve a sufficiently large safety region for controlling their postural stability. An everyday example of such task prioritization processes would be a child who stops talking to a friend while crossing a busy street intersection on his bicycle. 


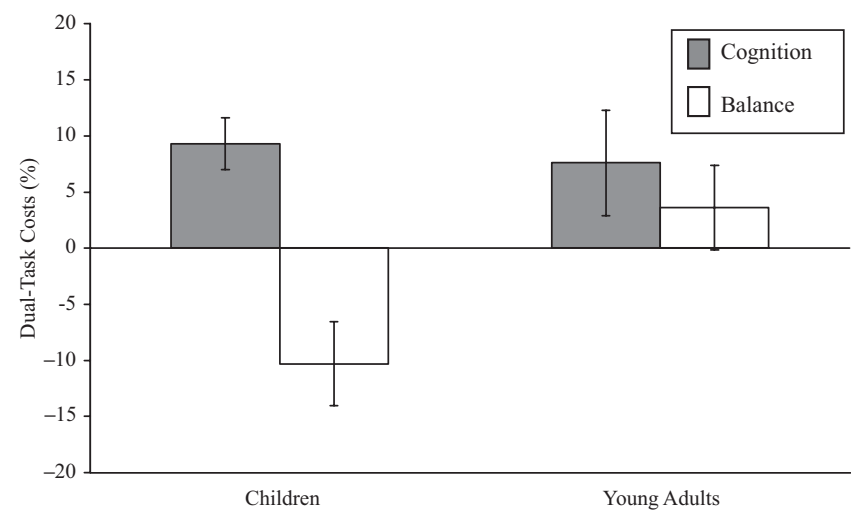

Figure 2. Children showed a trade-off pattern between cognitive and balance dual-task costs (DTCs); young adults did not. Positive DTC values indicate that performance deteriorated from single- to dual-task conditions, while negative values represent performance improvements made under dual-task conditions. Data were averaged across the two children's groups $(N=18)$ and across balance difficulty and cognitive task. Error bars represent standard errors of the mean.

Such behavior is adaptive, first, because it protects children from falls and their potentially harmful consequences, and second, because it allows them to safely negotiate resource constraints during concurrent task performance. Similar patterns of motor task prioritization have been observed in older adults (Li et al., 2001; Rapp et al., 2006). Our results also match findings demonstrating developmental phases of temporary overcontrol of posture during childhood (Hay \& Redon, 1999; Kirshenbaum, Riach, \& Starkes, 2001).

Young adults, on the other hand, who showed less body sway than children when balancing on the board, can "afford" to increase their sway under dual-task conditions without risking their balance. They therefore showed dual-task costs that were comparably high between the two task domains. However, under differential-emphasis conditions, young adults' performance did not vary significantly by task instruction. This pattern does not have to be interpreted as an inability to shift attention according to the instructions, since it could also be due to a ceiling effect. As can be seen in Figure 3, young adults performed at a very high level in that session, and they showed a trend toward performance improvements in the emphasized task domain.

It may be argued that findings on the automation of sensorimotor skills provide an alternative explanation of the present findings. Performance on well-practiced sensorimotor tasks often deteriorates when attention is focused on task execution (Beilock, Carr, MacMahon, \& Starkes, 2002; Maylor \& Wing, 1996; Swan, Otani, Loubert, Sheffert, \& Dunbar, 2004). In line with those considerations, a recent study by Huxhold, Li, Schmiedek, and Lindenberger (2006) found a U-shaped relationship between body sway and cognitive load for older adults, with increasing sway when there was no load or when cognitive load was very high and no increase in body sway for young adults with increasing cognitive

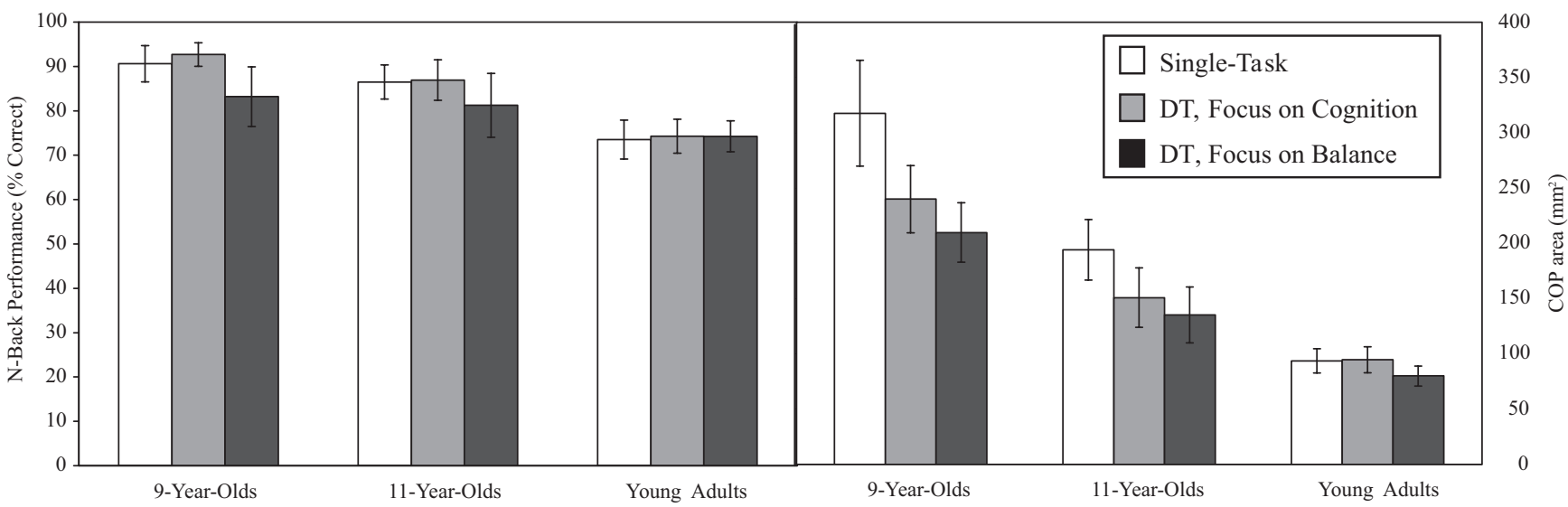

Cognitive Task

Balance Task

Figure 3. N-back performance did not vary systematically by instruction. Children continued to show smaller center of pressure (COP) areas in the dual-task situation even when they were instructed to focus on the cognitive task. Error bars show standard errors of the mean. DT = dual-task performance. 
load (see also Lövdén, Schaefer, Pohlmeyer, \& Lindenberger, in press). Apparently, focusing one's attention exclusively on an automated motor task can actually harm skillful task performance. However, it is hard to see why children would automate a motor skill, while young adults would not. Thus, automatization does not provide a convincing explanation of the observed age differences in task trade-offs. In addition, balancing under single-task conditions was always accompanied by a very easy secondary cognitive task (listening to animal voices or reading out numbers), such that attention was never focused exclusively on balancing. Finally, the differential-emphasis phase showed that body sway could be reduced when attention was focused on balancing. In sum, we consider it unlikely that interference with automatized behavior provides a compelling reason for why children prioritized balance performance more than adults did.

Motivational preferences for one task over the other also seem to have played a negligible role since all participants reported that they had focused more strongly on the cognitive task in the uninstructed dual-task situation. In addition, the order in which tasks were assessed within the dual-task sessions is unlikely to have influenced the pattern of findings, even though the dual-task trials were always assessed in the middle of the session, potentially leading to a greater influence of practice and fatigue on the single-task trials.

It could be surprising that balance-task difficulty (i.e., whether participants were balancing on the stable or on the moving platform) did not systematically influence the dual-task costs. A more difficult task should require more resources and thus lead to more pronounced performance decrements when resources have to be shared between two tasks (e.g., Guttentag, 1989; Wickens, 1984, 1991). However, the overall level of DTCs in the present study was not influenced by the difficulty of the balance task. Also, the trade-off pattern in children's DTCs did not become more pronounced when balancing on the moving rather than on the stable platform, although this should also have posed a greater challenge to their postural stability. A potential explanation is that the kinds of resources required to perform the two tasks concurrently originate from different and distinguishable resource pools (Navon \& Gopher, 1979) instead of a unique, general-purpose unit or structure (Kahneman, 1973). In this vein, the distinction between feedforward and feedback control of balance proposed by Hay and Redon (1999) could elucidate the processes required when balancing on the stable or moving platform. According to that account, feedforward control comes into play when an individual's own movements generate balance disturbances, and "anticipatory" postural adjustments can be triggered prior to the self-induced disturbance. Balancing on the ankle-disc board on the stable platform could represent such a situation, as an individual's own body movements generate all the sway. Feedback control, on the other hand, is relevant when movements are externally triggered and unpredictable and require a reaction of the body. This could occur when participants are balancing on the moving platform because they are forced to react to "external" platform tilt by shifting their weight. Perhaps, then, the amount of cognitive control required to keep one's balance is just as large or even larger when balancing on the stable platform, since feedforward control processes require a plan about the future state of the system for successful anticipatory movement regulation. Feedback control, on the other hand, is more reflexlike and does not require much cortical involvement.
The present study was able to show that children tend to prioritize the motor task when their bodily equilibrium is challenged in a demanding dual-task situation and that they continue to reduce their body sway under dual-task conditions even when they are instructed to focus more strongly on the cognitive task. This pattern of resource allocation is interpreted in terms of adaptive resource allocation. Young adults did not prioritize one task domain over the other, presumably because they did not have to do so. Future work should include situations in which young adults' balance is also challenged, probably resulting in task prioritization processes in this age group as well.

Another aspect that could enrich future research on cognitive and motor dual tasks is an extension of the study of taskcoordination processes to both ends of the life span through testing age groups within the same paradigm. In a recent study, Krampe, Schaefer, Lindenberger, and Baltes (2007) had children, young and older adults perform a word fluency task while they were walking a narrow track. Results showed that all age groups reduced their walking speed, but only the youngest children showed reliable dual-task costs in the cognitive domain. Since the motor task of walking on a narrow track did not prove an immediate threat to their balance, participants seem to have sacrificed their walking speed in order to keep up their cognitive performance.

In addition, it would be interesting to investigate the differential influences and interactions of intensive training with maturational or senescent changes in different samples. For example, researchers could make use of testing-the-limits methodologies (Kliegl, Smith, \& Baltes, 1989, 1990; Lindenberger \& Baltes, 1995) and investigate whether training is most efficient when the participants are trained for component tasks individually or under dual-task conditions (Pellechia, 2005). In this context, the systematic investigation of the influence of feedback and reinforcement on dualtask performances might add to our understanding of the unconscious or intentional nature of resource allocation processes at various ages.

Adaptive resource allocation in multi- or dual-task situations is an important topic in developmental research. The present study suggests that children prioritize a motor task when their postural stability is challenged. However, unless it results in more permanent bodily damage, going beyond one's equilibrium boundaries (e.g., falling) may have beneficial long-term ontogenetic consequences and lead to a greater range and proficiency of balancing behaviors, particularly in children. Future studies should try to identify the conditions under which children take greater balancing risks, focusing on both harmful and beneficial consequences for cognitive and motor development (cf. Diamond, 2000).

\section{References}

Baltes, P. B. (1987). Theoretical propositions of life-span developmental psychology: On the dynamics between growth and decline. Developmental Psychology, 23, 611-626.

Baltes, P. B. (1997). On the incomplete architecture of human ontogeny: Selection, optimization, and compensation as foundation of developmental theory. American Psychologist, 52, 366-380.

Baltes, P. B., \& Baltes, M. M. (1990). Psychological perspectives on successful aging: The model of selective optimization with compensation. In P. B. Baltes \& M. M. Baltes (Eds.), Successful aging: Perspectives from the behavioral sciences (pp. 1-34). New York: Cambridge University Press. 
Baltes, P. B., Lindenberger, U., \& Staudinger, U. M. (2006). Life span theory in developmental psychology. In R. M. Lerner (Ed.), Handbook of child psychology: Theoretical models of human development (6th ed., Vol. 1, pp. 569-664). New York: Wiley.

Beilock, S. L., Carr, T. H., MacMahon, C., \& Starkes, J. L. (2002). When paying attention becomes counterproductive: Impact of divided versus skill-focused attention on novice and experienced performance of sensorimotor skills. Journal of Experimental Psychology: Applied, 8, 6-16.

Birch, L. L. (1978). Baseline differences, attention, and age differences in time-sharing performance. Journal of Experimental Child Psychology, 25, 505-513.

Bower, G. H. (1970). Analysis of a mnemonic device. American Scientist, 58, 496-510.

Brehmer, Y., Li, S.-C., Müller, V., von Oertzen, T., \& Lindenberger, U. (2007). Memory plasticity across the life span: Uncovering children's latent potential. Developmental Psychology, 43, 465-478.

Brown, L. A., Sleik, R. J., Polych, M. A., \& Gage, W. H. (2002). Is the prioritization of postural control altered in conditions of postural threat in younger and older adults? Journals of Gerontology, Series A: Medical Sciences, 57, M785-M792.

Case, R., Kurland, D. M., \& Goldberg, J. (1982). Operational efficiency and the growth of short-term memory span. Journal of Experimental Child Psychology, 33, 386-404.

Craik, F. I. M., \& Bialystok, E. (2006). Cognition through the lifespan: Mechanisms of change. Trends in Cognitive Sciences, 10, 131-138.

DeOreo, K. D., \& Wade, M. G. (1971). Dynamic and static balancing ability of preschool children. Journal of Motor Behavior, 3, 326-335.

Diamond, A. (2000). Close interrelation of motor development and cognitive development and of the cerebellum and prefrontal cortex. Child Development, 71, 44-56.

Dobbs, A. R., \& Rule, B. G. (1989). Adult age differences in working memory. Psychology and Aging, 4, 500-503.

Fry, A. F., \& Hale, S. (1996). Processing speed, working memory, and fluid intelligence: Evidence for a developmental cascade. Psychological Science, 7, 237-241.

Guttentag, R. E. (1989). Age differences in dual-task performance: Procedures, assumptions, and results. Developmental Review, 9, 146-170.

Harnishfeger, K. K., \& Bjorklund, D. F. (1990). Children's strategies: A brief history. In D. F. Bjorklund (Ed.), Children's strategies: Contemporary views of cognitive development (pp. 1-22). Hillsdale, NJ: Erlbaum.

Hay, L., \& Redon, C. (1999). Feedforward versus feedback control in children and adults subjected to a postural disturbance. Experimental Brain Research, 125, 153-162.

Hitch, G. J., Towse, J. N., \& Hutton, U. (2001). What limits children's working memory span? Theoretical accounts and applications for scholastic development. Journal of Experimental Psychology: General, 130, $184-198$.

Huang, H-J., \& Mercer, V. S. (2001). Dual-task methodology: Applications in studies of cognitive and motor performance in adults and children. Pediatric Physical Therapy, 13, 133-140.

Huxhold, O., Li, S.-C., Schmiedek, F., \& Lindenberger, U. (2006). Dualtasking postural control: Aging and the effects of cognitive demand in conjunction with focus of attention. Brain Research Bulletin, 69, 294305.

Irwin-Chase, H., \& Burns, B. (2000). Developmental changes in children's abilities to share and allocate attention in a dual task. Journal of Experimental Child Psychology, 77, 61-85.

Kahneman, D. (1973). Attention and effort. Englewood Cliffs, NJ: Prentice-Hall.

Kail, R. (1991). Developmental change in speed of processing during childhood. Psychological Bulletin, 109, 490-501.

Kirshenbaum, L. A., Riach, C. L., \& Starkes, J. L. (2001). Non-linear development of postural control and strategy use in young children: A longitudinal study. Experimental Brain Research, 140, 420-431.

Kliegl, R., \& Lindenberger, U. (1993). Modeling intrusions and correct recall in episodic memory: Adult age differences in encoding of list context. Journal of Experimental Psychology: Learning, Memory, and Cognition, 19, 617-637.

Kliegl, R., Smith, J., \& Baltes, P. B. (1989). Testing-the-limits and the study of adult age differences in cognitive plasticity of a mnemonic skill. Developmental Psychology, 25, 247-256.

Kliegl, R., Smith, J., \& Baltes, P. B. (1990). On the locus and process of magnification of age differences during mnemonic training. Developmental Psychology, 26, 894-904.

Krampe, R. T., \& Baltes, P. B. (2003). Intelligence as adaptive resource development and resource allocation: A new look through the lenses of SOC and expertise. In R. J. Sternberg (Ed.), The psychology of abilities, competencies, and expertise (pp. 31-68). New York: Cambridge University Press.

Krampe, R. T., Schaefer, S., Lindenberger, U., \& Baltes, P. B. (2007). Life span dynamics of resource-allocation in multitask contexts: Concurrent walking and memory retrieval in children, young, and older adults. Manuscript submitted for publication.

Li, K. Z. H., Krampe, R. T., \& Bondar, A. (2005). An ecological approach to studying aging and dual-task performance. In R. W. Engle, G. Sedek, U. von Hecker \& D. N. McIntosh (Eds.), Cognitive limitations in aging and psychopathology (pp. 190-218). New York: Cambridge University Press.

Li, K. Z. H., Lindenberger, U., Freund, A. M., \& Baltes, P. B. (2001). Walking while memorizing: Age-related differences in compensatory behavior. Psychological Science, 12, 230-237.

Lindenberger, U., \& Baltes, P. B. (1995). Testing-the-limits and experimental simulation: Two methods to explicate the role of learning in development. Human Development, 38, 349-360.

Lindenberger, U., Kliegl, R., \& Baltes, P. B. (1992). Professional expertise does not eliminate age differences in imagery-based memory performance during adulthood. Psychology and Aging, 7, 585-593.

Lindenberger, U., Marsiske, M., \& Baltes, P. B. (2000). Memorizing while walking: Increase in dual-task costs from young adulthood to old age. Psychology and Aging, 15, 417-436.

Lippens, V. (2005, October). Can reading facilitate balancing on the gyro? Poster presented at the 11th Congrès International de l'Association des Chercheurs en Activitiés Physiques et Sportives (ACAPS) Conference, French Society for Sport and Movement Sciences, Paris, France.

Lövdén, M., Schaefer, S., Pohlmeyer, A. E., \& Lindenberger, U. (in press). Walking variability and working memory load in aging: A dual-process account relating cognitive control to motor control performance. Journals of Gerontology, Series B: Psychological Sciences and Social Sciences.

Lövdén, M., Schellenbach, M., Grossmann-Hutter, B., Krüger, A., \& Lindenberger, U. (2005). Environmental topography and postural control demands shape aging-associated decrements in spatial navigation performance. Psychology and Aging, 20, 683-694.

Manckoundia, P., Pfitzenmeyer, P., d'Athis, P., Dubost, V., \& Mourey, F. (2006). Impact of cognitive task on the posture of elderly subjects with Alzheimer's disease compared to healthy elderly subjects. Movement Disorders, 21, 236-241.

Marchese, R., Bove, M., \& Abbruzzese, G. (2003). Effect of cognitive and motor tasks on postural stability in Parkinson's disease: A posturographic study. Movement Disorders, 18, 652-658.

Maylor, E. A., \& Wing, A. M. (1996). Age differences in postural stability are increased by additional cognitive demands. Journals of Gerontology, Series B: Psychological Sciences and Social Sciences, 51, P143-P154.

Navon, D. (1984). Resources: A theoretical soup stone? Psychological Review, 91, 216-234. 
Navon, D., \& Gopher, D. (1979). On the economy of the human-processing system. Psychological Review, 86, 214-255.

Pellechia, G. L. (2005). Dual-task training reduces impact of cognitive task on postural sway. Journal of Motor Behavior, 37, 239-246.

Rapp, M. A., Krampe, R. T., \& Baltes, P. B. (2006). Adaptive task prioritization in aging: Selective resource allocation to postural control is preserved in Alzheimer's disease. American Journal of Geriatric Psychiatry, 14, 52-61.

Safe Kids Worldwide/Safe Kids U.S.A. (2007) Falls fact sheet. Retrieved March 12, 2008, from http://www.usa.safekids.org/content_documents/ 2007_Fact_SheetFalls.doc

Schaefer, S. (2005). Concurrent cognitive and sensorimotor performance: A comparison of children and young adults (Doctoral thesis, Free University Berlin, Germany). Available online at http://www.diss .fu-berlin.de/2005/178

Schaefer, S., Huxhold, O., \& Lindenberger, U. (2006). Healthy mind in healthy body? A review of sensorimotor-cognitive interdependencies in old age. European Review of Aging and Physical Activity, 3, 45-54.

Sheldon, J. H. (1963). The effect of age on the control of sway. Gerontologia Clinica, 5, 129-138.

Shumway-Cook, A., Woollacott, M., Kerns, K. A., \& Baldwin, M. (1997). The effects of two types of cognitive tasks on postural stability in older adults with and without a history of falls. Journals of Gerontology, Series A: Medical Sciences, 52, M232-M240.

Singer, T., Lindenberger, U., \& Baltes, P. B. (2003). Plasticity of memory for new learning in very old age: A story of major loss? Psychology and Aging, 18, 306-317.

Smith, E. E., \& Jonides, J. (1999, March 12). Storage and executive processes in the frontal lobes. Science, 283, 1657-1661.
Somberg, B. L., \& Salthouse, T. A. (1982). Divided attention abilities in young and old adults. Journal of Experimental Psychology: Human Perception and Performance, 8, 651-663.

Swan, L., Otani, H., Loubert, P. V., Sheffert, S., \& Dunbar, G. L. (2004). Improving balance by performing a secondary cognitive task. British Journal of Psychology, 95, 31-40.

Tewes, U. (Ed.). (1991). Hamburg-Wechsler-Intelligenztest für Erwachsene: Handbuch und Testanweisung [Hamburg-Wechsler intelligence test for adults: Handbook and manual]. Bern, Germany: Huber.

Tewes, U., Rossmann, P., \& Schallberger, U. (Eds.). (1999). HamburgWechsler-Intelligenztest für Kinder, dritte Auflage [Hamburg-Wechsler intelligence test for children, 3rd ed.]. Bern, Germany: Huber.

Wickens, C. D. (1980). The structure of attentional resources. In R. Nickerson (Ed.), Attention and performance VIII (pp. 239-257). Hillsdale, NJ: Erlbaum.

Wickens, C. D. (1984). Processing resources in attention. In R. Parasuraman \& D. R. Davies (Eds.), Varieties of attention (pp. 63-101). New York: Academic Press.

Wickens, C. D. (1991). Processing resources and attention. In D. Damos (Ed.), Multiple-task performance (pp. 3-34). London: Taylor-Francis.

Woollacott, M. H., \& Shumway-Cook, A. (1990). Changes in posture control across the life span: A systems approach. Physical Therapy, 70, 799-807.

Received September 22, 2006 Revision received November 26, 2007 Accepted January 10, 2008 